\title{
GIỚI THIỆU ỨNG DỤNG KẾT HỢP VIỄN THÁM VÀ MÔ HÌNH WATEM TRONG NGHIÊN CÚU XÓI MÒN ĐÂT KHU VỰC MIỀN NÚI
}

\author{
TS. PHẠM MINH HẢl, TS. VŨ KIM CHI, CN. NGUYẼN MINH NGỌC \\ Viện Khoa học Đo đạc và Bản đồ
}

\section{Tóm tắt:}

Hiện nay đã có nhiều nghiên cứu được phát triển để lượng hóa hiện tượng xói mòn đất trên đất dốc tại Việt Nam. Các phương pháp tính toán xói mòn đất truyền thống dựa trên các phương pháp đo đạc thực địa trên các rãnh chứa nước và sau đó dùng phương pháp nội suy cho các khu vực liền kề. Việc đo đạc lượng xói mòn trên một điểm sử dụng những phương pháp này mất nhiều thời gian và độ chính xác của kết quả nội suy sự phân bố hiện tượng xói mòn đất trên toàn vùng chưa thống nhất. Việc nghiên cứu phát triển một cách tiếp cận mới có thể cung cấp thông tin trực quan, định lượng và định tính về hiện tượng xói mòn đất là điều hết sức cần thiết. Trong phạm vi bài báo này, nhóm tác giả giới thiệu kết quả nghiên cứu ứng dụng viễn thám và phần mềm WATEM trong nghiên cứu xói mòn đất khu vực miền núi, với khu vực thử nghiệm tại huyện Mường La, tỉnh Sơn La.

\section{Giới thiệu chung}

Việt Nam là nước nhiệt đới gió mùa, khí hậu và địa hình phân hóa phức tạp. Hiện tượng xói mòn, đặc biệt ở khu vực có độ dốc cao, xảy ra phổ biến và đa dạng gây nguy hại làm cho đất mất dần các chất dinh dưỡng dẫn tới suy thoái gây tác hại nghiêm trọng cho sản xuất nông nghiệp và môi trường sinh thái.

Hiện nay đã có nhiều nghiên cứu được phát triển để lượng hóa hiện tượng xói mòn đất trên đất dốc tại Việt Nam. Các phương pháp tính toán xói mòn đất truyền thống dựa trên các phương pháp đo đạc thực địa trên các rãnh chứa nước và sau đó dùng phương pháp nội suy cho các khu vực liền kề. Việc đo đạc lượng xói mòn trên một điểm sử dụng những phương pháp này mất nhiều thời gian và độ chính xác của kết quả nội suy sự phân bố hiện tượng xói mòn đất trên toàn vùng chưa thống nhất. Bên cạnh các phương pháp truyền thống, các mô hình nghiên cứu hiện tượng xói mòn đất hiện nay sử phương pháp tính toán xói mòn được đưa ra dựa trên học thuyết xói mòn được phát triển bởi Ellision (1944).
Phương trình xói mòn bao gồm 4 yếu tố: loại đất sử dụng, sườn dốc, mặt độ che phủ đất, lượng mưa. Trong đó lượng mưa là thành phần ảnh hưởng đến xói mòn. Tuy nhiên, phương pháp tính xói mòn này được phát triển dựa trên phương pháp phân loại theo kiểu hiện tượng xói mòn đất, dữ liệu đưa ra được thể hiện trong bảng thống kê. Do vậy, việc nghiên cứu phát triển một cách tiếp cận mới có thể cung cấp thông tin trực quan, định lượng và định tính về hiện tượng xói mòn đất là điều hết sức cần thiết.

Trong bài báo này, tác giả giới thiệu kết quả nghiên cứu ứng dụng viễn thám và phần mềm WATEM trong nghiên cứu xói mòn đất khu vực miền núi, với khu vực thử nghiệm tại huyện Mường La, tỉnh Sơn La với diện tích $1 \mathrm{~km}^{2}$. Không giống các mô hình trước đây (như WEPP hoặc EUROSEM), mô hình WATEM tập trung vào định lượng, và mô tả phân bố không gian của hiện tượng xói mòn dựa trên các tham số đầu vào như bản đồ lớp phủ mặt đất, bản đồ sông suối. 

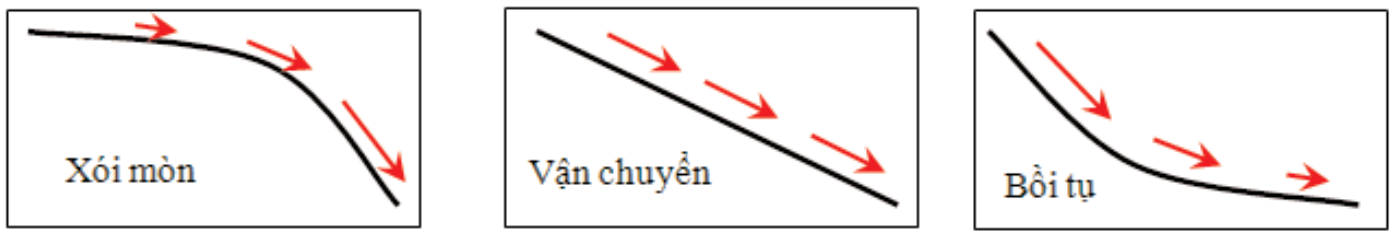

Hình 1: Mô tả các quá trình xói mòn và bồi tụ mô hình sử dụng

2. Cơ sỏ' khoa học của mô hình WATEM/SEDEM

Mô hình WATEM được phát triển bởi nhóm các nhà nghiên cứu địa vật lý Trường Đại học K.U.Leuven, Vương quốc Bỉ. Các cơ chế xói mòn và bồi tụ của mô hình (hình 1) được phát triển với mục đích để tính toán lượng xói mòn đất và lắng đọng trầm tích. (Van Rompaey và ctv, 2001).

Mô hình sử dụng RUSLE được phát triển bởi Renard và ctv (1997) để tính toán xói mòn đất.

$$
E=R \times K \times L S_{2 D} \times C \times P
$$

Trong đó:

E: lượng đất mất đi $\left(\mathrm{kg} \mathrm{m}^{-2} \mathrm{yr}^{-1}\right)$

R: lượng mưa (MJ $\mathrm{mm} \mathrm{m}^{-2} \mathrm{~h}^{-1} \mathrm{yr}^{-1}$ )

$\mathrm{K}$ : yếu tố thổ nhưỡng $\left(\mathrm{kg} \mathrm{h} \mathrm{MJ}^{-1} \mathrm{~mm}^{-1}\right)$

C: hoạt động canh tác

$P$ : yếu tố kiểm soát xói mòn đất
$\mathrm{LS}_{2 \mathrm{D}}$ : yếu tố chiều dài sườn dốc địa hình, được tính theo phương trình của Desmet và Goyer (1996) cho địa hình trong không gian 2 chiều.

$$
L S_{i, j}=\frac{\left(A_{i, j}+D^{2}\right)^{m+1}-A_{i, j}^{m+1}}{D^{m+2} x^{m}{ }_{i, j}(22.13)^{m}}
$$

Trong đó:

$L S_{i, j}$ là chiều dài sườn dốc địa hình lưới với tọa độ $(\mathrm{i}, \mathrm{j})$,

Ai,j-in là diện tích khu vực tính toán trong trong lưới tọa độ $(\mathrm{i}, \mathrm{j})(\mathrm{m} 2)$,

$D$ là độ dài lưới $(m)$

$x_{i, j}=\sin \lambda_{i, j}+\cos \lambda_{i, j}$ với $\lambda_{i, j}$ là điểm lưới với tọa độ $(\mathrm{i}, \mathrm{j})$

m: tham số độ dốc. (Xem hình 2)

Với lượng trầm tích được vận chuyển xuống lòng sông suối hướng ra cửa sông, quá trình vận chuyển trầm tích dựa trên khả

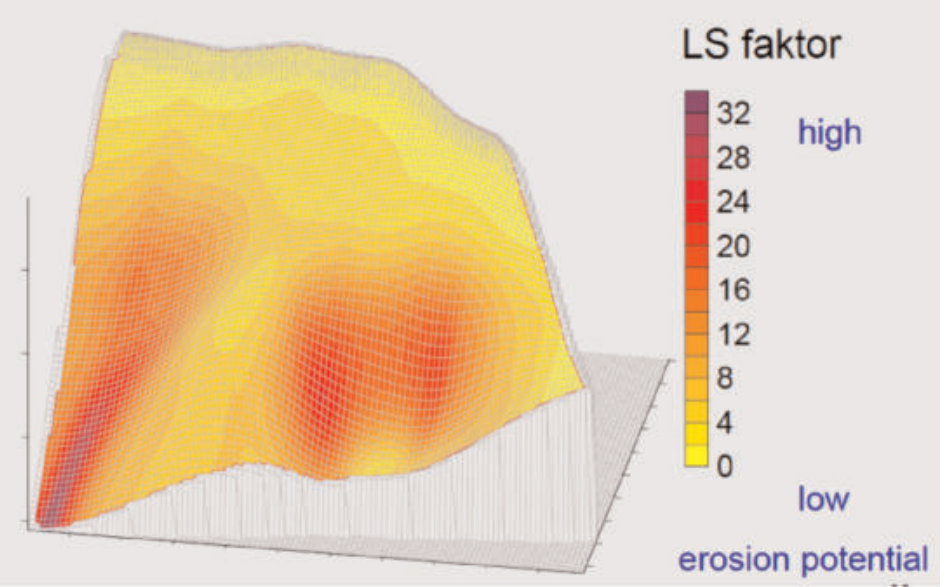

Hình 2: Ví dụ về sự liên quan giữa yếu tố LS với khả năng xói mòn đất (Kritof, 2004) 
năng vận chuyển của dòng chảy, được tính toán như sau (Van Rompaey và ctv, 2001):

$$
T C=K_{T C} \times R \times K \times\left(L S_{2 D}-4 \times I S^{0.8}\right)
$$

Trong đó:

TC: khối lượng vật chất bị vận chuyển $\left(\mathrm{kg} \mathrm{m}^{-1} \mathrm{yr}^{-1}\right)$,

$\mathrm{K}_{\mathrm{TC}}$ : hệ số vận chuyển

S: giá trị độ dốc $(\mathrm{m} / \mathrm{m})$

Bên cạnh đó, mô hình cũng sử dụng phương pháp đã được phát triển bởi
Govers (1991) để tính toán lượng đất bồi tụ:

$$
T C=k T C \times \text { Erill }
$$

Trong đó:

kTC là mối liên hệ tới thực phủ

$$
\begin{aligned}
& \text { Erill }=\text { Epot }- \text { Eirill } \\
& \text { Erill }=R K C \times(4 \times \text { slope }) \\
& \text { Slope: độ dốc sườn núi }
\end{aligned}
$$

Tính toán được Epot được tóm tắt bằng sơ đồ sau (hình 3): (Xem hình 3)

\subsection{Dữ liệu đầu vào}

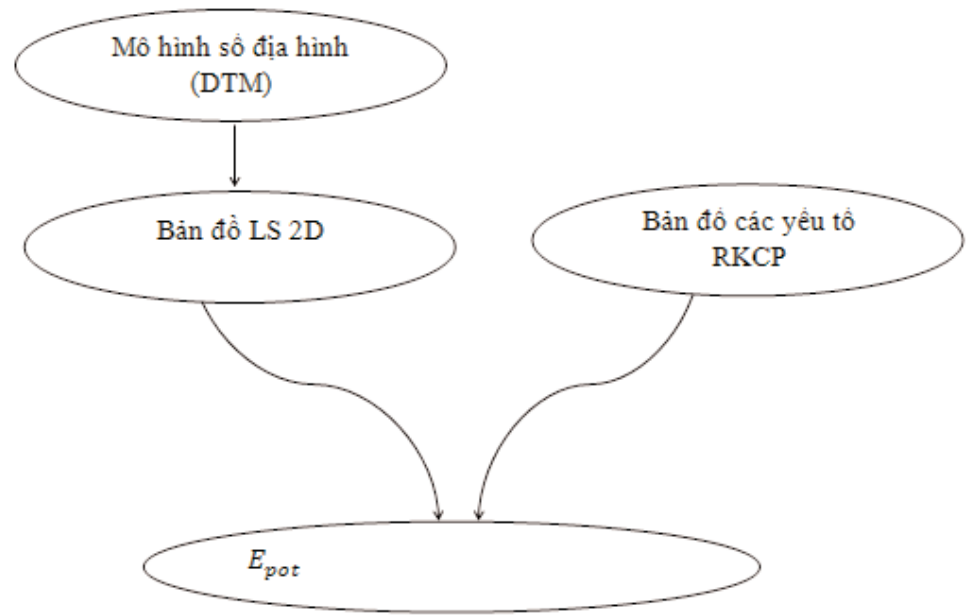

Hình 3: Quy trình tính $E_{\text {pot }}$ (Kristof,2014)

2.1.1. Mô hình số độ cao (Yếu tố chính )

Nhóm nghiên cứu lựa chọn khu vực thử nghiệm của nghiên cứu này thuộc địa phận huyện Mường La, tỉnh Sơn La. Phần mềm WATEM sử dụng mô hình số độ cao để tạo vùng và tính toán độ dốc địa hình. Chất lượng của kết quả đầu ra phụ thuộc rất lớn vào độ chính xác của mô hình số độ cao, với giá trị độ cao tăng dần, không có vùng giá trị 0. Trong nghiên cứu dưới đây, sử dụng DEM tỷ lệ $1 / 50000$ để đánh giá vùng nghiên cứu với khoảng cao đều $25 \mathrm{~m}$.

2.1.2. Bản đồ sử dụng đất (Yếu tố chính)
Trong bài báo này, nhóm tác giả sử dụng ảnh vệ tinh SPOT 5 chụp năm 2013 khu vực Sơn La thành lập bản đồ lớp phủ mặt đất khu vực nghiên cứu. Phương pháp phân loại không kiểm định được sử dụng để chiết xuất thông tin thành lập bản đồ sử dụng đất. Các lớp thông tin sau phân loại ảnh lại geocode theo các giá trị yêu cầu của mô hình WATEM:

Lớp sông: -1

Đất nông nghiệp: 10

Rừng: 10.000 
Hinh
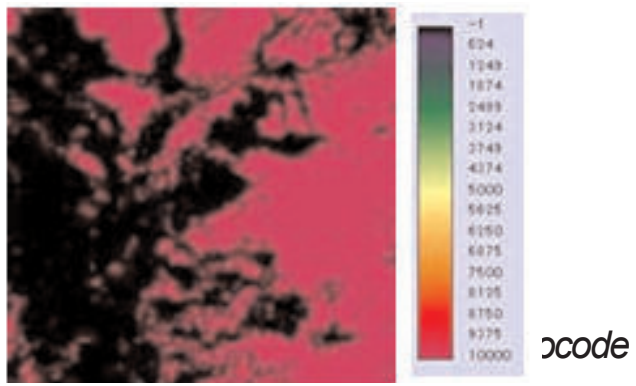

2.1.3. Lớp sông suối (Yếu tố chính)

2.1.4. Yếu tố thổ nhưỡng (K) (Yếu tố phụ)

Yếu tố thổ nhưỡng được tính theo công thức sau (Romkens và ctv, 1986)

(5)

$$
K=0.035+0.038 \exp \left[-0.5 \times\left(\frac{\log D_{g}+1.519}{0.7584}\right)\right]
$$

Yếu tố thổ nhưỡng chịu ảnh hưởng và liên quan chặt chẽ bởi khả năng thấm nước, cấu trúc, độ tợ xốp, sức liên kết giữa các hạt đất. Kích thước đường kính của hạt đất được xác định bởi $D_{g}(\mathrm{~mm})$ (Shirazi và

Boursma, 1984) như sau:

$$
D_{g}=\exp \left[\sum_{i} 0.01 x f_{i} \ln \left(m_{i}\right)\right]
$$

$f_{i}$ là tỷ lệ phần trăm kích thước hạt

$\mathrm{m}_{\mathrm{i}}$ là trung bình cộng của các giới hạn kích thước hạt (mm).

$$
E_{l}=2.03\left[1+0.19 \cos \left(2 \pi f_{i}-\omega\right)\right] \sum_{k=1}^{N} R_{k}^{1.4}
$$

\subsubsection{Yếu tố mưa và dòng chảy mặt $(R)$}

Yếu tố mưa được tính toán theo công thức sau (Yu và Rosewell, 1996)

Trong đó $\mathrm{R}_{\mathrm{k}}$ lượng mưa trung trình ngày $\left(\mathrm{R}_{\mathrm{k}}>12,7 \mathrm{~mm}\right)$ và $\mathrm{N}$ là số ngày mưa với lượng mưa trung bình, $f_{i}$ là hệ số $\left(f_{i}=1 / 12\right)$ được sử dụng để mô tả tự thay đổi theo mùa.

Sự phân bố của mùa mưa cũng là yếu tố chi phối và quyết định đến lượng đất mất do xói mòn. Những trận mưa lớn nếu xảy ra ở những thời điểm đất trông trải cũng là nguyên nhân làm cho lượng đất bị mất nhiều hơn.

\subsubsection{Yếu tố canh tác $(P)$}

Với mô hình WATEM, nhóm tác giả đặt mặc định $\mathrm{C}$ là 0,37 . Nghiên cứu giả định một ô vuông diện tích đất canh tác vận chuyển 3,3 lần lượng trầm tích hơn một ô lưới dưới rừng và đồng cỏ.

Yếu tố này chỉ ra mức độ tác động của các hệ thống cây trồng và những khác biệt trong quản lý sử dụng đất đối với lượng đất bị mất do xói mòn. Các rừng và đồng cỏ là những hệ thống bảo vệ đất tự nhiên tốt nhất, sau đó là các loại cây trồng có khả năng che phủ cao thường được trồng mật độ dày.

\section{Kết quả}

Mô hình WATEM được ứng dụng để tính toán lượng đất xói mòn cho khu vực nghiên cứu. Kết quả sau xử lý là các bản đồ như sau: Bản đồ yếu tố LS, bản đồ hướng dòng chảy, Bản đồ bồ tụ, Bản đồ xói mòn do dòng chảy bề mặt. Ở đó, các giá trị trong bản đồ độ dốc thể hiện các góc độ dốc định hướng dòng chảy. Giá trị chiều dài độ dốc được dùng để tính toán tỷ lệ xói mòn do dòng chảy. Góc dốc định hướng xói mòn tuyến tính. Tỷ lệ bồi tụ thể hiện đơn vị tấn/ha. Nếu điểm ảnh có giá trị âm trên bản đồ, đó sẽ là khu vực bị xói mòn. Nếu điểm ảnh có giá trị dương thì điểm ảnh đó thể hiện vùng bồi tụ trầm tích.

Kết quả thu được từ mô hình WATEM không chỉ sự phân bố không gian mà còn định lượng được khối lượng xói mòn và bồi tụ. Tổng hợp bản đồ xói mòn và bồi tụ khu 

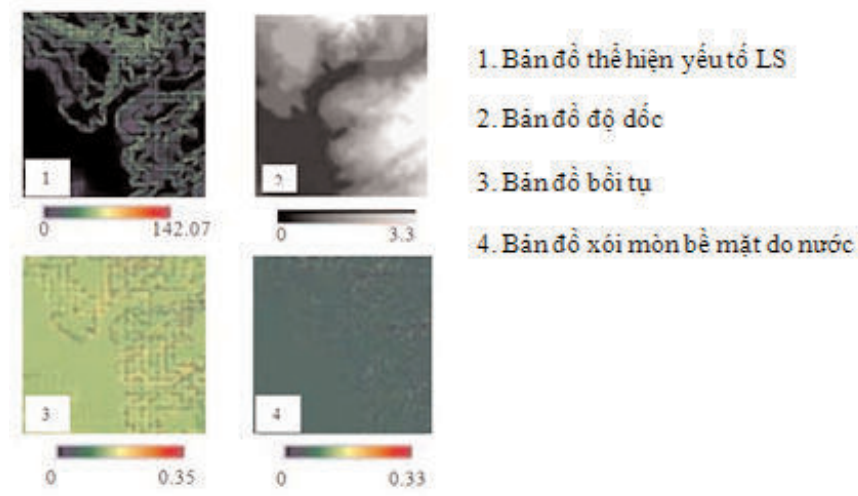

Hình 5: Các kết quả đầu ra mô hình WATEM
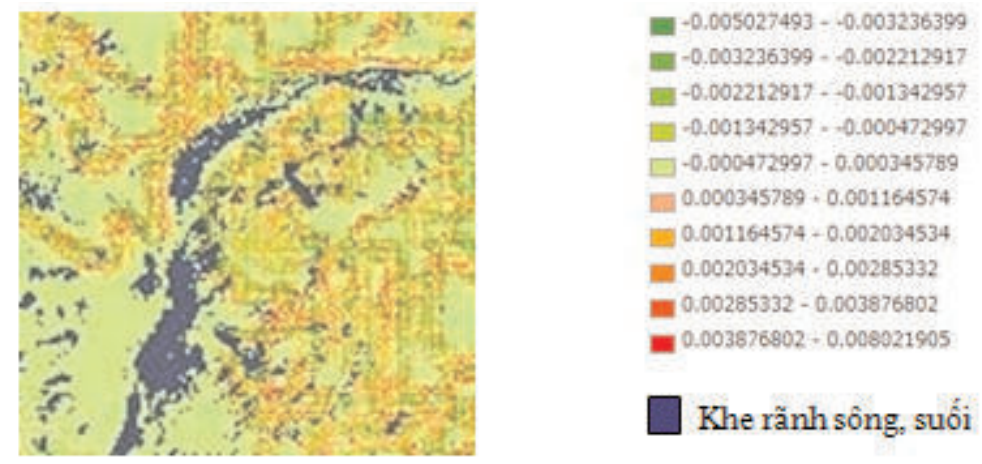

Hình 6: Bản đồ xói mòn và bồi tụ khu vực thử nghiệm

vực thử nghiệm thể hiện trên hình 6 . Qua kết quả tính toán, lượng đất bị xói mòn của khu vực thử nghiệm là 0,64 tấn/năm. (Xem hình 6)

\section{Kết luận}

Kết hợp dữ liệu viễn thám mô hình WATEM trong mô phỏng hiện tượng xói mòn và bồi tụ khu vực thử nghiệm không chỉ cung cấp những thông tin trực quan mà còn định lượng được lượng xói mòn và bồi tụ của khu vực. Tuy nhiên, chất lượng kết quả đầu ra phụ thuộc nhiều vào chất lượng của $D E M$ và độ phân giải của các file dữ liệu raster đầu vào. Việc sử dụng dữ liệu ảnh viễn thám đã phát huy được vai trò là dữ liệu gốc để thành lập bản đồ lớp phủ mặt đất, giúp mô hình WATEM có thể đáp ứng được khả năng giám sát hiện tượng xói mòn đất trên diện rộng, đặc biệt ở khu vực miền núi. $O$

\section{Tài liệu tham khảo}

[1]. Clement A. Okia (2002). Global Perspectives on Sustainable Forest Management. Chapter: Deforestation: Causes, Effects and Control Strategies, trang16.

[2]. Desmet, P.J.J. and Govers, G. (1996). A GIS-procedure for automatically calculating the USLE LS-factor on topographically complex landscape units. Journal of Soil and Water Conservation, 51 (5), trang 427-433.

[3]. Ellison, W. D. (1944). Studies of raindrop erosion. Agriculture and Engineering, 25 
(6), trang 131.

[4]. Govers, G. (1991). Rill erosion on arable land in Central Belgium: rates, controls and predictability. Catena, 18, trang 133-155.

[5]. Kristof, 2014. Spatial modeling \& Soil Conservation Strategies at the landscape level. IAEA Workshop 28-31 October 2014, Shanghai, China.

[6]. Nguyễn Kim Lợi, 2005. Lecture in soil erosion estimation (in Vietnamese).

[7]. Renard, K.G., Foster, G.R., Weesies, G.A., Yoder, D.C. (1997). Preidcting soil erosion by water: A guide to conservation planning with the Revised Universal Soil Loss Equation (RUSLE). Agriculture Handbook No.703.

[8]. Römkens, M.J.M. (1985). The soil erodibility factor: a perspective. In: ElSwaify, S.A., Moldenhauer, W.C., Lo, A. (Eds.), Soil Erosion and Conservation. Soil Conservation Society of America, Ankeny, trang 445-461.

[9]. Shirazi, M and Boersma, L. (1984). A unifying quantitative analysis of soil texture. Soil Science Society of America Journal. 48, trang 142

[10]. Van Oost K, Govers, G. and Desmet, P.J.J. (2000). Evaluating the effects of changes in landscape structure on soil erosion by water and tillage. Landscape Ecology 15 (6), trang 579-591.

[11]. Van Rompaey, A., Verstraeten, G., Van Oost K, Govers, G. and Poesen, J. (2001). Modelling mean annual sediment yield using a distributed approach. Earth Surface Processes and Landforms, 26 (11), trang 1221-1236.

[12]. Verstraeten, G., Van Oost K, Van Rompaey, A., Poesen, J. and Govers, G. (2002). Evaluating an integrated approach to catchment management to reduce soil loss and sediment pollution through modelling. Soil Use and Management, 18, trang 386-394.

[13]. Yu, B and Rosewell, C,J. (1996). An assessement of a daily trainfall erosivity model for new south wales. Australia Journal of Soil Research, 34, trang 139. 0

\section{Summary}

\section{Introduction of remote sensing applications and WATEM model in study of soil}

\section{erosion in mountainous areas}

Dr. Pham Minh Hai, BSc. Nguyen Minh Ngoc, Institute of Geodesy and Cartography

Dr. Vu Kim Chi, Institute of Vietnamese Studies and Development Sciences

Recently, there are some studies developed to quantify soil erosion on sloping land in Vietnam. The traditional methods of calculating soil erosion based on field measurements on the water storage and then used interpolation method for the adjacent area become popular. The soil erosion measurement in a site using these above methods takes a lot of time and the accuracy of interpolated results of soil erosion distributions across the region is inconsistent. Therefore, it is essential to develop a new approach which can provide with visual, quantitative and qualitative information of soil erosion, especially for sloping land in Vietnam. In this paper, authors introduce an approach using remote sensing data and WATEM model to quantify soil erosion with the study area located in Muong La district, Son La province.

Ngày nhận bài: 20/02/2015. 